American Journal of Applied Sciences 4 (2): 85-88, 2007

ISSN 1546-9239

(C) 2007 Science Publications

\title{
Design and Simulation of an Absorption Diffusion Solar Refrigeration Unit
}

\author{
B. Chaouachi and S. Gabsi \\ Research Unit: Environment, Catalysis and Analysis of Processes \\ National School of Engineers of Gabès (E.N.I.G)- Omar Ibn ElKhattab Street -6029 Gabès- \\ Tunisia.
}

\begin{abstract}
The purpose of this study was the design and the simulation of an absorption diffusion refrigerator using solar as source of energy, for domestic use. The design holds account about the climatic conditions and the unit cost due to technical constraints imposed by the technology of the various components of the installation such as the solar generator, the condenser, the absorber and the evaporator. Mass and energy conservation equations were developed for each component of the cycle and solved numerically. The obtained results showed, that the new designed mono pressure absorption cycle of ammonia was suitable well for the cold production by means of the solar energy and that with a simple plate collector we can reach a power, of the order of 900 watts sufficient for domestic use.
\end{abstract}

Key words: Solar energy, simulation, absorption refrigeration, mono pressure, design

\section{INTRODUCTION}

If the solar energy possesses the advantage to be "clean", free and renewable, this last is probably, considered like an adapted potential solution, that answers in even time at a economic preoccupation and ecological problems.

Among the main done currently research is the use of this free source to make operate system of refrigeration.

The diffusion absorption refrigerator cycle invented in the 1920s is based on ammonia (refrigerant) and water (absorbent) as the working fluids together with hydrogen as an auxiliary inert gas ${ }^{[1,2]}$. Since there are no moving parts in the unit, the diffusion absorption refrigerator system is both quiet and reliable. The system is, therefore, often used in hotel rooms and offices $^{[3]}$.

The absorption diffusion refrigerating machine is designed according to the operation principle of the refrigerating machine mono pressure invented by PLATERN and MUNTER ${ }^{[2-10]}$. This machine uses three operation fluids, water (absorbent), the ammonia (refrigerant) and hydrogen as an inert gas used in order to maintain the total pressure constant. The simplest configuration of the designed machine, represented on Fig. 1, is composed of the principal following elements:

* An evaporator

* A condenser

* An absorber

* A heat exchanger

* Solar collector

* Rectifier
* Separator

Under operation the rich solution water-ammonia, coming from the storage tank, passes in the tubes of the solar collector (1), where it will be heated by the solar flux in this collector. A vaporization of ammonia is produced in this tubes and the product vapor take the poor solution to the separator.

The generated ammonia vapor containing a small water vapor quantity will be purified in the rectifier. The condensate water vapor returns in the separator (6) whereas the ammonia vapor penetrates in the condenser (7) in which it is liquefied. After that the liquid ammonia passes from the condenser to the evaporator (8) where it comes into contact with a hydrogen gas flux (9) facilitating its evaporation by absorbing a quantity of heat from the medium to be cooled. The mixture gas of hydrogen-ammonia (11), which produced at the evaporator temperature, comes into indirect contact with the poor solution, coming from the separator, in the heat exchanger where it absorbs a quantity of heat of the latter. At the exit exchanger (13), the cooled weak solution penetrates in the absorber where it absorbs ammonia of the ammonia-hydrogen gas mixture. This absorption is favored at low temperature and it will be almost total. Insoluble hydrogen in water goes up in top of the storage tank (9) and returns in the evaporator whereas the rich solution water-ammonia enters in the solar collector tubes and a new cycle start again.

Theoretical analyzes: The evaluation of the refrigerating machine performance criteria requires the knowledge of momentum, energy and mass balance. The liquid and vapor flow rates are determined by

\footnotetext{
Corresponding Author:

B. Chaouachi, Research Unit: Environment, Catalysis and Analysis of Processes. National School of Engineers of Gabès (E.N.I.G)- Omar Ibn ElKhattab Street -6029-Gabès-Tunisia. Tel: 0021675392 380, Fax: 0021675392190
} 
developing a bubble pump model based on the developed model in the literature ${ }^{[4,11 \text {, and } 12]}$.

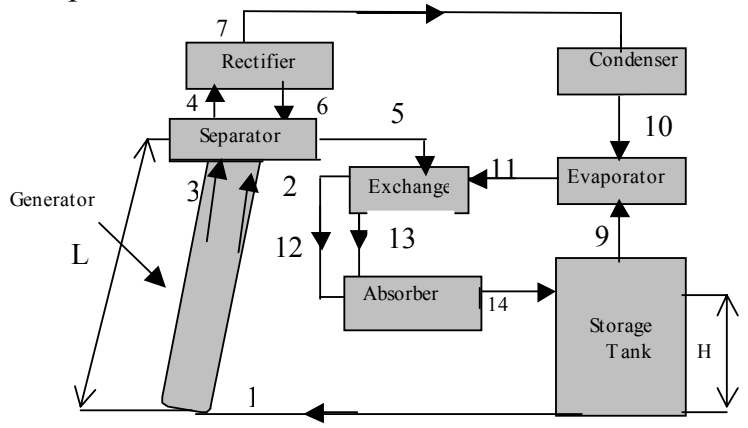

Fig. 1: Schematic diagram of the absorption diffusionrefrigerating machine

The coefficient of performance of this refrigerating machine with absorption diffusion is defined as follows:

$\mathrm{COP}=\frac{\mathrm{Q}_{\mathrm{E}}}{\mathrm{Q}_{\mathrm{G}}}$

Mass and energy balances: In this paragraph, we will have to establish the mass and energy balance equations for the various elements of the refrigerating cycle. The hydrogen-ammonia mixture is supposed an ideal mixture.

Bubble pump (Currents 1, 2 and 3):

General mass balance equation:

$\dot{m}_{3}+\dot{m}_{2}=\dot{m_{1}}$

Ammonia mass balance equation:

$\dot{m}_{3} \xi_{3}+\dot{m}_{2} \psi_{2}=\dot{m}_{1} \xi_{1}$

Energy balance

$-\dot{\mathrm{m}}_{1} \mathrm{~h}_{1}+\dot{\mathrm{m}}_{3} \mathrm{~h}_{3}+\dot{\mathrm{m}}_{2} \mathrm{~h}_{2}=\mathrm{Q}_{\mathrm{G}}$

Separator (Currents 2, 3, 4, 5 and 6):

General mass balance equation:

$\dot{\mathrm{m}}_{4}+\dot{\mathrm{m}}_{5}=\dot{\mathrm{m}}_{2}+\dot{\mathrm{m}}_{3}+\dot{\mathrm{m}}_{6}$

Ammonia mass balance equation:

$\dot{\mathrm{m}}_{5} \xi_{5}+\dot{\mathrm{m}}_{4} \psi_{4}=\dot{\mathrm{m}}_{3} \xi_{3}+\dot{\mathrm{m}}_{2} \psi_{2}+\dot{\mathrm{m}}_{6} \xi_{6}$

Energy balance

$\stackrel{\bullet}{-\mathrm{m}_{5}} \mathrm{~h}_{5}-\stackrel{\bullet}{\mathrm{m}}_{4} \mathrm{~h}_{4}+\stackrel{\bullet}{\mathrm{m}_{3}} \mathrm{~h}_{3}+\stackrel{\bullet}{\mathrm{m}_{2}} \mathrm{~h}_{2}+\stackrel{\bullet}{\mathrm{m}_{6}} \mathrm{~h}_{6}=0$

Rectifier (Currents 4, 6 and 7):

General mass balance equation:

$\dot{\mathrm{m}}_{6}+\dot{\mathrm{m}}_{7}=\dot{\mathrm{m}}_{4}$

Ammonia mass balance equation:

$\dot{\mathrm{m}}_{7}=\dot{\mathrm{m}}_{4} \psi_{4}$

Energy balance

$-\dot{\mathrm{m}}_{7} \mathrm{~h}_{7}-\dot{\mathrm{m}}_{6} \mathrm{~h}_{6}+\dot{\mathrm{m}}_{4} \mathrm{~h}_{4}=\mathrm{Q}_{\mathrm{R}}$
Condenser (Currents 7 and 8):

General mass balance equation:

$\dot{\mathrm{m}}_{7}=\dot{\mathrm{m}}_{8}$

Energy balance

$\dot{\mathrm{m}}_{7} \mathrm{~h}_{7}+\dot{\mathrm{m}}_{8} \mathrm{~h}_{8}=\mathrm{Q}_{\mathrm{C}}$

Evaporator (Currents 8, 9 and 11):

General mass balance equations:

$\dot{m}_{10}=\stackrel{\bullet}{m_{8}+} \dot{m}_{9}$
$\dot{m}_{10}=\dot{m}_{11}$

Ammonia mass balance equation:

$\dot{m}_{8}=\dot{m}_{11} \psi_{11, N H 3}$

Energy balance

$\dot{m}_{9}\left(h_{11, H}-h_{9}\right)+\dot{m}_{8}\left(h_{11, N H 3}-h_{8}\right)=Q_{E}$

Heat Exchanger (Currants 5, 11, 12 et 13):

General mass balance equation:

$\dot{m}_{12}+\dot{m}_{13}=\dot{m}_{11}+\dot{m}_{5}$

Ammonia mass balance equation:

$\dot{m}_{13} \xi_{13}=\dot{m}_{5} \xi_{5}$
$\stackrel{\bullet}{m_{11}} \psi_{11, N H 3}=\dot{m}_{12} \psi_{12, N H 3}=m_{11, N H 3}$

Energy balance

$\dot{m}_{11, H}\left(h_{12, H}-h_{11, H}\right)+\dot{m}_{11, N H 3}\left(h_{12, N H 3}-h_{11, N H 3}\right)=Q_{12-11}$

$m_{5}\left(h_{13}-h_{5}\right)=Q_{5-13}$

Absorber (Currents 12, 13 and 14):

General mass balance equation:

$\dot{\mathrm{m}}_{12}+\dot{\mathrm{m}}_{13}=\dot{\mathrm{m}}_{14}$

Ammonia mass balance equation:

$\dot{\mathrm{m}}_{12} \psi_{12}+\dot{\mathrm{m}}_{13} \xi_{13}=\dot{\mathrm{m}}_{14} \xi_{14}$

Water mass balance equation:

$\dot{m}_{14}\left(1-\xi_{14}\right)-\dot{m}_{9}=\dot{m}_{13}\left(1-\xi_{13}\right)$

Energy balance

$$
\begin{aligned}
& \dot{m}_{13} h_{13}+\left(\dot{m}_{12}-\dot{m}_{9}\right) h_{12, N H 3}-\left(\dot{m}_{14}-\dot{m}_{9}\right) h_{14} \\
& +\dot{m}_{11, H}\left(h_{12, H}-h_{14, H}\right)=Q_{A}
\end{aligned}
$$

Storage tank (Currents 1, 9 and 14):

General mass balance equation:

$\dot{m}_{9}+\dot{m}_{1}=\dot{m_{14}}$

Ammonia mass balance equation:

$\dot{m}_{14} \xi_{14}=\dot{m}_{1} \xi_{1}$

Energy balance

$\left(\dot{m_{14}}-\dot{m}_{9}\right)\left(h_{14}-h_{1}\right)+\dot{m}_{9}\left(h_{14}-h_{9}\right)=0$ 


\section{RESULTS AND DISCUSSION}

The operating boundaries of the system were examined by conducting simulations for various values of the generator temperature, $\mathrm{T}_{\mathrm{G}}$, the evaporator temperature, $T_{E}$, the pressure of the system, $\mathrm{P}$ and the generator heat input, $\tilde{\mathrm{Q}_{\mathrm{G}}}$. The operation ranges were found to be:

$5<\mathrm{P}<15$ bar, $330<\mathrm{T}_{\mathrm{G}}<410 \mathrm{~K}, 263<\mathrm{T}_{\mathrm{E}}<283 \mathrm{~K}$. The obtained results were showed in figures.

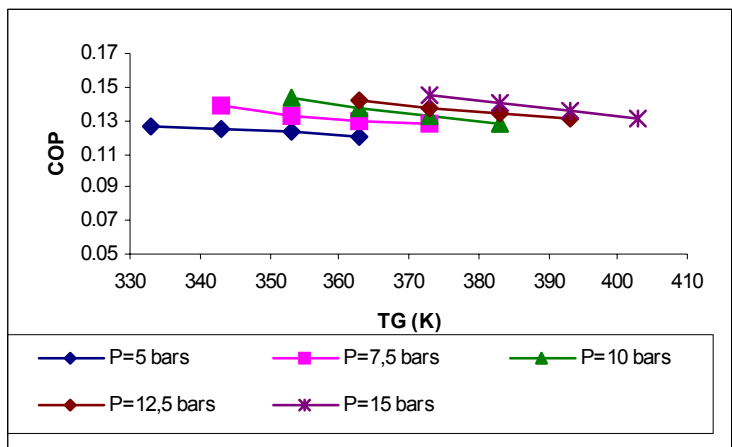

Fig. 2: $\mathrm{COP}$ vs. to generator temperature for various pressures of the system $(\xi \mathrm{r}=0.45, \mathrm{Te}=273 \mathrm{~K})$

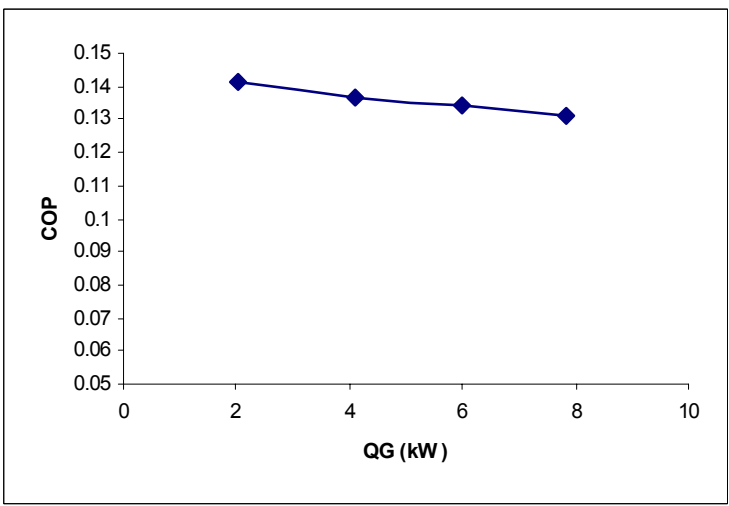

Fig. 3: OP vs. to generator power $(\xi \mathrm{r}=0.45$, $\mathrm{Te}=273 \mathrm{~K}, \mathrm{P}=12.5$ bars)

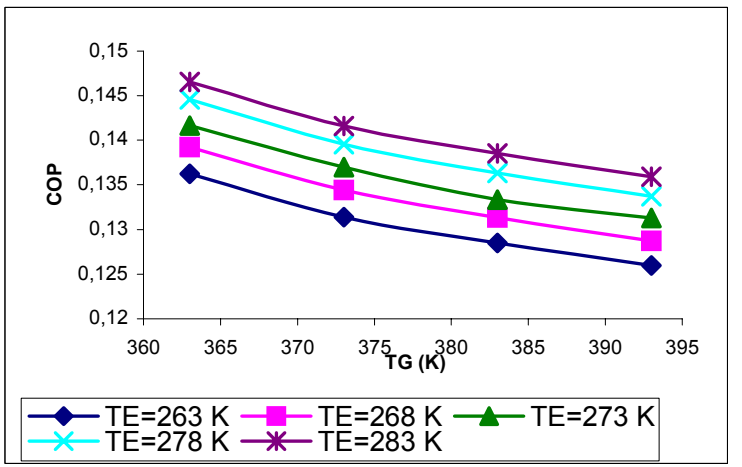

Fig. 4: $\mathrm{OP}$ vs. the evaporator temperature $(\xi \mathrm{r}=0.4$, $\mathrm{P}=12.5$ bars, $\mathrm{H} / \mathrm{L}=0.6$ )

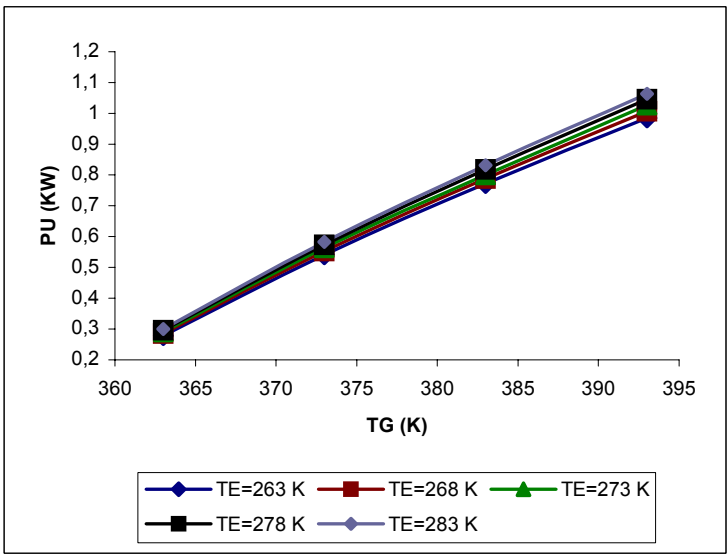

Fig. 5: $\mathrm{Q}_{\mathrm{E}}$ vs. to the evaporator temperature $(\xi \mathrm{r}=0.4$, $\mathrm{P}=12.5$ bars, $\mathrm{H} / \mathrm{L}=0.6$ )

Figure 2 presents the COP vs. the generator temperature for different pressures of the system for a fixed rich concentration and evaporator temperature. It shows that the COP decreases as the generator temperature increases and it increases when the pressure increases too. This is may be explained by the fact that a smaller amount of ammonia was separated from the ammonia-water solution and thus more solution had to be circulated so as to maintain the refrigerant flow rate in the condenser. It thus recommended that pressure of the system as high as possible.

The dependence of COP on the generator input at fixed rich concentration, evaporator temperature and pressure of the system shows that the COP decreases when the generator heat input increases (Fig. 3). This is may be explained by the same manner have Fig. 2 .

The Fig. 4 shows that the COP decreases as the generator temperature increases. It was also found that the higher the evaporator temperature, the higher COP, i. e. that more heat was absorbed in the evaporator. There are thus opposing demands for the evaporator temperature; on the one hand, it should be high enough (depending on the desired cooling capacity) to yield a higher COP.

An investigation of dependence of $\mathrm{Q}_{\mathrm{E}}$ on the generator temperature for different evaporator temperatures at fixed pressure and rich concentration showed that $\mathrm{Q}_{\mathrm{E}}$ increased as the generator temperature. This is can be explained by the fact that more quantity of ammonia was generated when the generator temperature increases.

\section{CONCLUSION}

A design and simulation of an absorption diffusion refrigerator using solar as source of energy, for domestic use was done and the system performances were analyzed parametrically by computer simulation. 
It appears that best performance in terms of COP would be obtained when we work with low generator temperature and high pressure. In the other hand, the values of COP remain weak and depend of the power solar babble pump.

\author{
Nomenclature \\ COP: coefficient of performance \\ Q: heat transfer power $(\mathrm{J} / \mathrm{s})$ \\ h: enthalpy $(\mathrm{kJ} / \mathrm{kg})$ \\ $\mathrm{H} / \mathrm{L}$ : submergence \\ $\mathrm{m}$ : mass flow $(\mathrm{kg} / \mathrm{s})$ \\ P: $\quad$ pressure (bar) \\ $\mathrm{T}: \quad$ temperature $(\mathrm{K})$ \\ $\xi$ : mass fraction of cooling agent in the solution \\ $\psi: \quad$ mass fraction of cooling agent in a mixture gas
}

\section{Subscripts}

$1,2,3 \ldots$ system's point designation

A: Absorber

C: Condenser

E: Evaporator

G: Generator

H: Hydrogen

$\mathrm{NH}_{3}$ : Ammonia

R: Rectifier

r: rich

\section{REFERENCES}

1. Srikhirin, P. and S. Aphornratana, 2002. Investigation of a diffusion absorption refrigerator. Appl. Therm. Engg., 22: 1181-1193.

2. Shelton, S.V., 1999. Design analysis of the Einstein refrigeration cycle. Ph. D. Georgia Institute of Technology, USA.

3. De Francisco, A., R. Illanes, J.L. Torres, M. Castillo, M. De Blas, E. Prieto and A. Garcia, 2002. Development and testing of a prototype of low power water-ammonia absorption equipment for solar energy applications. Renewable Energy, 25: 537-544.
4. Koyfman, A., M. Jelinek, A. Levy and I. Borde, 2003. An experimental investigation of bubble pump performance for diffusion absorption refrigeration system with organic working fluids. Appl. Therm. Engg., 23: 1881-1894.

5. Mejbri, K.H., N. Ben Ezzine, Y.Guizani and A. Bellagi, 2005. Discussion of the feasibility of the Einstein refrigeration cycle. Intl. J. Refrigeration, 29: 60-70.

6. Florides, J.A., S.A. Kalogirou, S.A. Tassou and L.C. Wrobel, 2003. Design and construction of LiBr-water absorption machine. Energy Conversion and Management, 44: 2483-2508.

7. Srikhirin, P., S. Aphormatana and S. Chungpaibulpatana, 2001. A review of absorption refrigeration technologies. Renewable and Sustainable Energy Reviews, 5: 343-372.

8. kang, Y.T., Y. Kunugi and T. Kashiwagi, 2000. Review of advanced absorption cycles: Performance improvement and temperature lift enhancement. Intl. J. Refrigeration, 23: 388-401.

9. Chekir, N., K. Mejbri and A. Bellagi, 2006. Simulation d'une machine frigorifique a' absorption fonctionnant avec des mélanges d'alcanes. Intl. J. Refrigeration, 29: 469-475.

10. Zohar, A., M. Jenilik and A. Levy, 2005. Numerical investigation of a diffusion absorption refrigeration cycle. Intl. J. Refrigeration, 28 : 515525 .

11. Collier, J.G., 1982. Convective Boiling and Condensation. Mc Graw Hill International Book Company, pp: 26-58.

12. Stewart, S.W., 2001. Bubble Pump Design and Performance. Thesis Presented to the Academic Faculty, Georgia Institute of Technology, USA. 